\title{
Ground calibration of the high-sensitivity SELENE lunar magnetometer LMAG
}

\author{
Hisayoshi Shimizu ${ }^{1}$, Futoshi Takahashi², Naoki Horii ${ }^{2}$, Ayako Matsuoka $^{3}$, \\ Masaki Matsushima ${ }^{2}$, Hidetoshi Shibuya ${ }^{4}$, and Hideo Tsunakawa ${ }^{2}$ \\ ${ }^{1}$ Earthquake Research Institute, University of Tokyo, Tokyo, Japan \\ ${ }^{2}$ Department of Earth and Planetary Sciences, Tokyo Institute of Technology, Tokyo, Japan \\ ${ }^{3}$ Institute of Space and Astronautical Science, Japan Aerospace Exploration Agency, Kanagawa, Japan \\ ${ }^{4}$ Department of Earth Sciences, Kumamoto University, Kumamoto, Japan
}

(Received March 26, 2007; Revised August 14, 2007; Accepted October 31, 2007; Online published April 9, 2008)

\begin{abstract}
Ground calibration experiments of the SELENE high sensitivity fluxgate Lunar Magnetometer (LMAG) have been performed in order to determine the alignment, sensitivity, and offset of the sensors (MGF-S). It is checked out that the sensors are orthogonal to each other within 0.4 degrees, and the linearity of the ambient magnetic field and the output from the sensors are confirmed. Also, the temperature dependences of the offset and sensitivity are examined but no clear signatures of temperature dependencies can be seen. SELENE has an in-flight calibration system in order to determine the direction of the magnetometer routinely. The magnetic fields generated by the sensor alignment monitor coil (SAM-C) system are used for the in-flight calibration. The magnetic field distributions generated by SAM-C are determined and the accuracy of determination of the magnetometer position and direction is estimated. Multiple measurements will allow us to determine the direction of MGF-S with about 0.1-degree accuracy. Appropriate corrections from the results of the ground and in-flight calibrations will allow us to recover the magnetic field near the moon with accuracy about $0.1 \mathrm{nT}$.
\end{abstract}

Key words: SELENE project, magnetometer, ground calibration, in-flight calibration.

\section{Introduction}

Observations of the lunar magnetic field have been conducted occasionally since 1969, from Apollo 12 to Lunar Prospector. Number of magnetic anomalies on the surface of the moon have been identified (e.g. Hood et al., 1979; Hood and Schubert, 1980; Halekas et al., 2001; Hood et al., 2001; Kurata et al., 2005; Toyoshima et al., 2008), though the moon does not have an intrinsic magnetic field at present. The cause of the magnetic anomalies are still in debate (see, e.g. Hood, 1987), and the question if the moon had generated its magnetic field in the past, probably right after the formation of the moon, still remains. Existence of a metallic core of substantial size is also an interesting question to discuss the origin of the moon and its history thereafter (e.g. Righter, 2002; also, Lee et al., 1997; Runcorn, 1996). The SELENE/Lunar Magnetometer (LMAG) team aims at clarifying above mentioned issues by accumulating magnetic signatures originated from the moon such as those magnetic anomalies on the surface and also by identifying time-dependent magnetic responses which have information on the electrical conductivity of the interior of the moon (e.g. Dyal et al., 1975, 1976; Russell et al., 1981; Hobbs, 1973; Hood et al., 1982, 1999).

The LMAG has a set of tri-axis ring-core type fluxgate sensors (MGF-S) to observe the magnetic field in orbit

Copyright (c) The Society of Geomagnetism and Earth, Planetary and Space Sciences (SGEPSS); The Seismological Society of Japan; The Volcanological Society of Japan; The Geodetic Society of Japan; The Japanese Society for Planetary Sciences; TERRAPUB. round the moon continuously during the project. The near polar orbit is designed at about $100 \mathrm{~km}$ altitude. Previous studies found that the magnetic field intensity of the $100 \mathrm{~km}$ scale magnetic anomalies observed at $100 \mathrm{~km}$ altitude is of order $0.1 \mathrm{nT}$. Also, accuracy of the same order of magnitude is required for the study of magnetic responses of the moon (e.g. Dyal et al., 1975). The LMAG must fulfill the requirement for successful observations and analyses.

Designed specifications of the LMAG are shown in Table 1, which are similar to those of the GEOTAIL magnetometer (Yamamoto et al., 1996). The noise level of the ring-core sensors is much less than $0.1 \mathrm{nT}$. Field measurements may be performed with either of the four ranges. range- 0 will be (automatically) selected in actual observations orbiting the moon. The LMAG has high resolution and low noise level which are enough to detect the magnetic field signature of the moon. The MGF-S will operate from the far end of the 12-meter mast extending from the main body of SELENE to avoid the interference magnetic fields due to magnetic matters and/or electric currents of the spacecraft and other scientific instruments. The SELENE project has strict electromagnetic compatibility (EMC) conditions that the effect of each of them at the position of the fluxgate sensor is less than $0.02 \mathrm{nT}$, and all the instruments and satellite are confirmed to fulfill the requirement through EMC tests.

It is essential to know the offset, sensitivity and relative directions (alignments) of each of the fluxgate sensors beforehand by proper calibrations in order to obtain magnetic 
Table 1. Specification of LMAG.

\begin{tabular}{ll}
\hline sensor & ring-core \\
\hline linearity & better than $10^{-3}$ \\
\hline dynamic range & range-3: $\pm 65536 \mathrm{nT}$ \\
& range- $: \pm 1024 \mathrm{nT}$ \\
& range-1 $: \pm 256 \mathrm{nT}$ \\
& range-0 $: \pm 64 \mathrm{nT}$ \\
\hline resolution & range-3:2.0 nT \\
(16 bit) & range-2 $: 0.03 \mathrm{nT}$ \\
& range-1 $: 0.008 \mathrm{nT}$ \\
& range-0 $: 0.002 \mathrm{nT}$ \\
\hline noise level & less than $0.1 \mathrm{nT}$ \\
\hline sampling rate & $32 \mathrm{~Hz}$ \\
\hline
\end{tabular}

field data accurately with appropriate corrections. Also, the temperature dependencies of the offset and sensitivity should be checked because the fluxgate systems, both the sensors and electronics, often show large temperature dependencies. Calibration experiments have been conducted and those quantities have been obtained. The setups of the experiments and the results are shown in Section 2.

Monitoring the alignment of the MGF-S with respect to spacecraft is also required to obtain a reliable magnetic field vector in the vicinity of the moon, and thus SELENE has an in-flight calibration system. This kind of system is necessary because the mast, on which MGF-S is installed, may be deformed more or less by thermal expansion if the satellite is in day or night side and the difference of the the relative position and angle of the magnetic sensors to the spacecraft may possibly be significant. Monitoring of the magnetometer alignment can be achieved if some known magnetic field is observable. Although the geomagnetic main field can be used for satellites orbiting the earth (Olsen et $a l ., 2003$ ) or passing nearby the earth for swingby (Anderson et al., 2001; Dougherty et al., 2004), it is necessary for SELENE to generate a known magnetic field by itself because it does not observe a known natural magnetic field. We have developed a sensor alignment monitor coil system (SAM-C) employing a bi-axis coil configuration in order to generate two linearly independent magnetic field. SAM-C is installed at the root of the mast, and it produces a magnetic field of about $2 \mathrm{nT}$ at the position of the MGF-S. The spatial distributions of the SAM-C generated magnetic field have been determined as magnetic potential distributions by ground experiments. The details of the experiment and the results are shown in Section 3. The accuracy of the determination of the magnetometer position and alignment using SAM-C generated magnetic field is estimated. The formulation and results are also shown in Section 3.

\section{Ground Calibration of LMAG}

\subsection{Sensitivity and alignment}

The principle of the ground calibration of magnetometer is fairly simple; applying a known magnetic field to the MGF-S and examining the output from it (e.g. Yamamoto et al., 1996; Lohr et al., 1997; Risbo et al., 2003; Dougherty et al., 2004). However, to control the magnetic environment and applying magnetic field and to monitor the relative alignment between the direction of MGF-S and given magnetic field need particular methods and equipments.

Calibration experiments had been carried out in the magnetic test facility in Tsukuba Space Center, NASDA (now, JAXA). The facility has a main 3-axis $15 \mathrm{~m}$ diameter Braunbek coil system to create almost zero-magnetic field environment by canceling the geomagnetic field and its disturbances. An equivalent Braunbek coil system, of which size is $1 / 10$ of the main coil system, is employed to detect the geomagnetic disturbances and to feedback the information to the main system. The combination of the coil systems can control the environment against more than $\pm 1000 \mathrm{nT}$ disturbances. The main coil system achieves magnetic environment of less than $2.5 \mathrm{nT}$ in the spherical space of $2.3 \mathrm{~m}$ diameter centering the coil system as a result.

We have followed the calibration method by Yamamoto et al. (1996) which determines the sensitivity and alignment of the magnetometer and also the direction of the known magnetic field simultaneously. Multiple configurations between the magnetometer and the coil system are employed in the method in order to determine the relative directions of the sensors and those of coil axes altogether because only one configuration is not sufficient as shown later. This was done by rotating the MGF-S around a sensor axis.

The known magnetic field is applied using a 3-axis Helmholtz coil system (calibration coil) which can produce a magnetic field within $0.05 \mathrm{nT}$ accuracy in each direction if nominal applied field is $30000 \mathrm{nT}$. Cubic mirrors are attached onto the MGF-S and calibration coil system in order to setup the experiments with high accuracy using a laser alignment system. Orthogonality of the surfaces of the cubic mirrors are essential to obtain the required relative directions accurately. The precision of the right angles between the surfaces of the cubic mirrors are better than 0.1 degrees, which is the required accuracy of the MGF-S sensors.

Now, the related parameters are the sensitivity of MGF$\mathrm{S}\left[A_{i}(i=x, y, z)\right]$ (nT/digit), output of the MGF-S $\left[M_{i}\right]$ (digit), applied magnetic field $\left[B_{i}\right](\mathrm{nT})$, the transform matrix from the alignment mirror coordinate attached on MGF$\mathrm{S}$ (orthogonal) to the sensor coordinate (may not be orthogonal) $\left[\mathbf{C}_{\epsilon}\right]$, the transform matrix from the coil system coordinate to the alignment mirror coordinate attached on the coil $\left[\mathbf{C}_{\delta}\right]$, the transform matrix from the alignment mirror coordinate of the coil to the alignment mirror coordinate of the sensor $[\mathbf{K}]$, and the zero-offsets $\left[B_{\text {offset }, i}\right]$ of the sensors. By using the quantities listed above, the relationship of them may be written as

$$
\left(\begin{array}{ccc}
A_{x} & 0 & 0 \\
0 & A_{y} & 0 \\
0 & 0 & A_{z}
\end{array}\right)\left(\begin{array}{c}
M_{x} \\
M_{y} \\
M_{z}
\end{array}\right)=\mathbf{C}_{\epsilon} \mathbf{K C}_{\delta}\left(\begin{array}{c}
B_{x} \\
B_{y} \\
B_{z}
\end{array}\right)+\left(\begin{array}{c}
B_{\text {offset }, x} \\
B_{\text {offset }, y} \\
B_{\text {offset }, z}
\end{array}\right),
$$

or

$$
\mathbf{A M}=\mathbf{C}_{\epsilon} \mathbf{K C}_{\delta} \mathbf{B}+\mathbf{B}_{\text {offset }} .
$$

Each sensor or coil direction may be expressed with two angles in corresponding mirror coordinate as shown in Fig. 1. Therefore, explicit forms of $\mathbf{C}_{\epsilon}$ and $\mathbf{C}_{\delta}$ are

$$
\mathbf{C}_{\epsilon}=\left(\begin{array}{ccc}
\cos \theta_{x} \cos \phi_{x} & \cos \theta_{x} \sin \phi_{x} & \sin \theta_{x} \\
\cos \theta_{y} \sin \phi_{y} & \cos \theta_{y} \cos \phi_{y} & \sin \theta_{y} \\
\cos \theta_{z} \sin \phi_{z} & \sin \theta_{z} & \cos \theta_{z} \cos \phi_{z}
\end{array}\right)
$$


a)

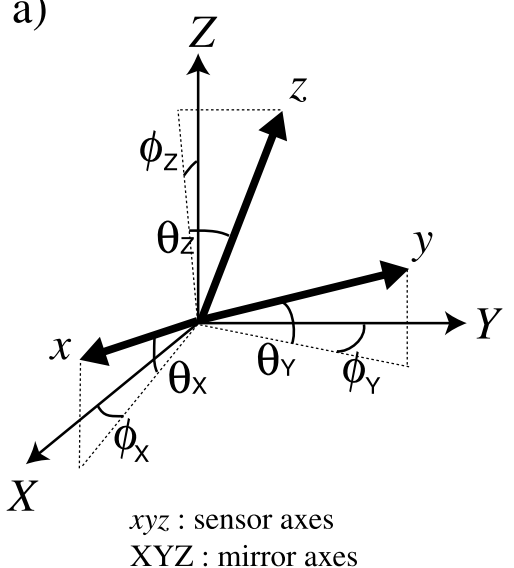

b)

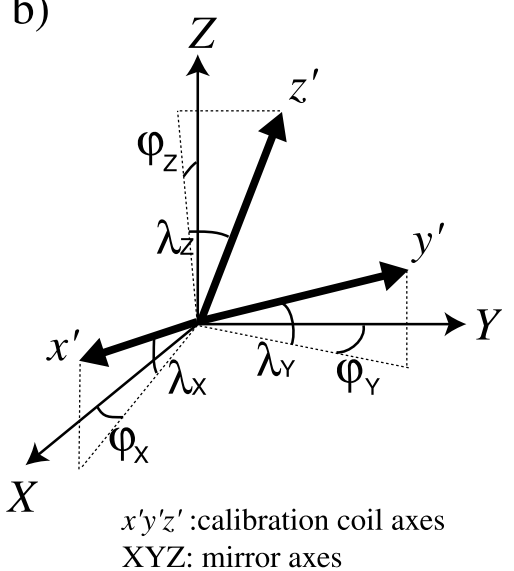

Fig. 1. Definitions of the angles. (a) Angles between the alignment mirror coordinate and the magnetic field sensor coordinate. (b) Angles between the alignment mirror coordinate and the calibration coil coordinate.

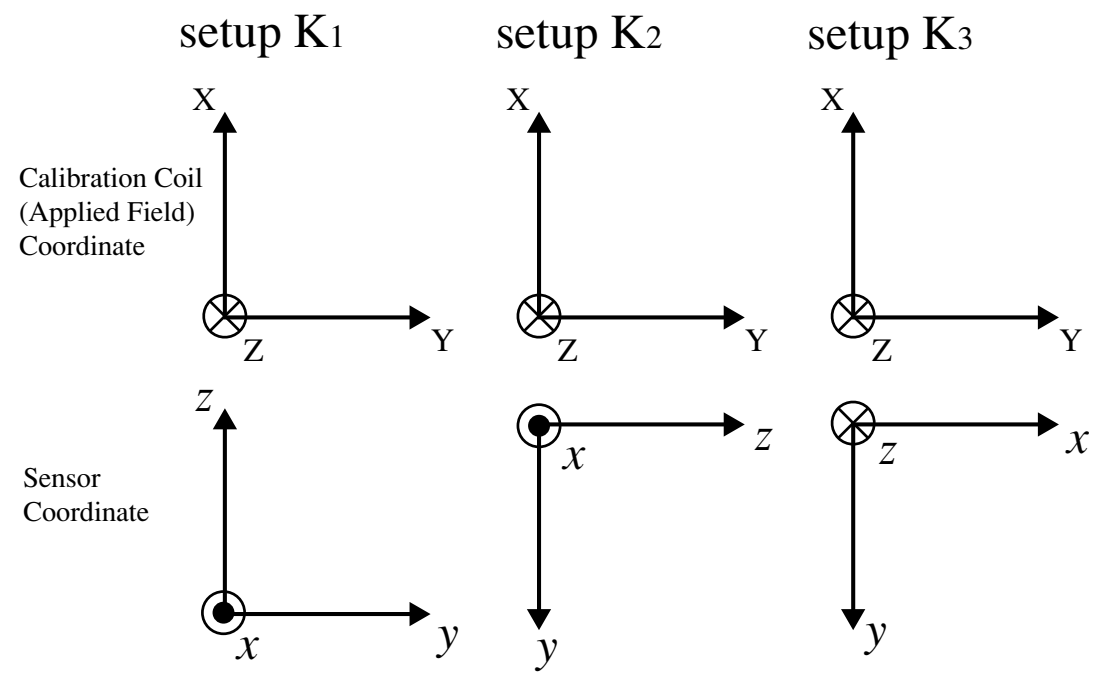

Fig. 2. Configurations of the coil and the sensor.

and

$$
\mathbf{C}_{\delta}=\left(\begin{array}{ccc}
\cos \lambda_{x} \cos \varphi_{x} & \cos \lambda_{y} \sin \varphi_{y} & \cos \lambda_{z} \sin \varphi_{z} \\
\cos \lambda_{x} \sin \varphi_{x} & \cos \lambda_{y} \cos \varphi_{y} & \sin \lambda_{z} \\
\sin \lambda_{x} & \sin \lambda_{y} & \cos \lambda_{z} \cos \varphi_{z}
\end{array}\right) .
$$

Note that $\mathbf{C}_{\epsilon}$ and $\mathbf{C}_{\delta}$ are very close to unit matrices.

In observation equation (2), magnetic field $\mathbf{B}$ and setup of the experiment, i.e., the relationship between the direction of the sensor and the coil in each mirror coordinate system $\mathbf{K}$ are given. Then, the output from the MGF-S, $\mathbf{M}$, is recorded. Intensity of the applied magnetic field is changed during the experiment for each setup. This allows us to eliminate the effect of the zero-offset $\mathbf{B}_{\text {offset }}$ in Eq. (2) and also to confirm the linearity of the output from the MGF-S to the applied magnetic field. Therefore, $\mathbf{B}, \mathbf{K}$ and $\mathbf{M}$ are used to determine components of $\mathbf{A}, \mathbf{C}_{\epsilon}$, and $\mathbf{C}_{\delta}$. There are 15 unknowns to be determined in total. In actual determination of the sensitivity of alignment, matrices in Eqs. (3) and (4) are represented as

$$
\mathbf{C}_{\eta}=\left(\begin{array}{ccc}
1+\eta_{x x} & \eta_{x y} & \eta_{x z} \\
\eta_{y x} & 1+\eta_{y y} & \eta_{y z} \\
\eta_{z x} & \eta_{z y} & 1+\eta_{z z}
\end{array}\right)
$$

Table 2. Applied field during the calibration experiments.

\begin{tabular}{ll}
\hline range & applied field (nT) \\
\hline range- 3 & $0, \pm 25000, \pm 50000$ \\
range- 2 & $0, \pm 300, \pm 600, \pm 900$ \\
renge-1 & $0, \pm 80, \pm 160, \pm 240$ \\
range- 0 & $0, \pm 10, \pm 20, \pm 40, \pm 60$ \\
\hline
\end{tabular}

where $\eta$ is $\epsilon$ or $\delta$. Six components are independent in $\mathbf{C}_{\eta}$ matrix because we have conditions that row (column) vectors in $\mathbf{C}_{\epsilon}\left(\mathbf{C}_{\delta}\right)$ are unit vectors. In this case, we have 21 unknowns with six conditions.

From one setup of the experiment, i.e. one $\mathbf{K}$ and $\mathbf{B}$ in the $x, y$, and $z$ directions, independently, we can obtain nine equations. If we rotate the magnetometer by 90 degrees with respect to one of the coordinate, we have nine more equations but only six are independent of the previous setup. In this case, we have 21 conditions to determine 21 unknowns. This is sufficient to determine the unknowns but the equations may not be solved stably due to the influence of measurement errors. Thus, we added one more setup, 

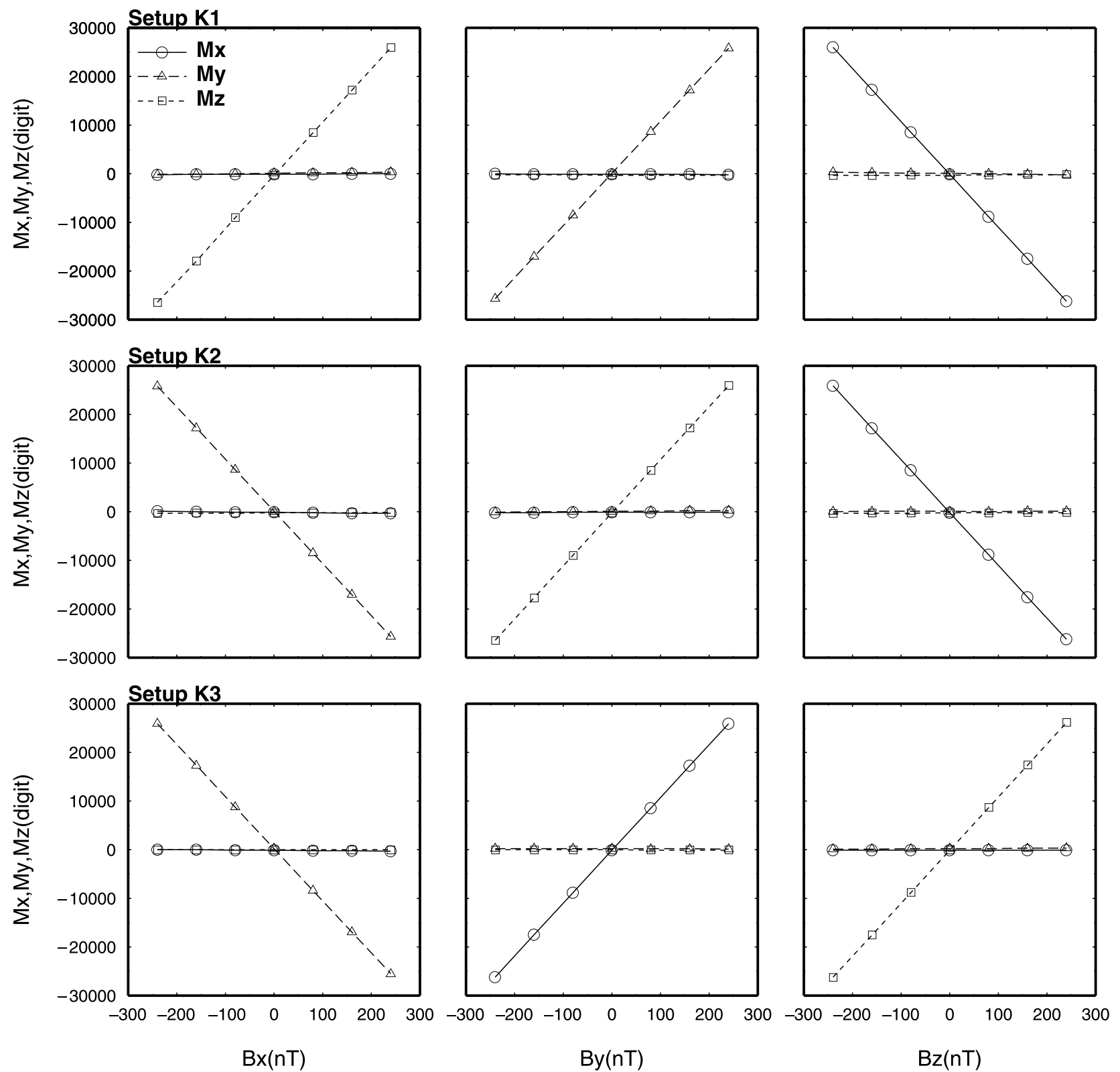

Fig. 3. Output $\mathbf{M}$ due to input $\mathbf{B}$ with range-1.

rotate the magnetometer by 90 degrees around another axis, and made measurements. The number of independent equations are now 27, and determination of the sensitivity and alignment becomes relatively easy and stable.

Setups of the measurements are shown in Fig. 2. The $\mathbf{K}$ matrices corresponding to the setups are the following;

$$
\begin{aligned}
\mathbf{K}_{1} & =\left(\begin{array}{rrr}
0 & 0 & -1 \\
0 & 1 & 0 \\
1 & 0 & 0
\end{array}\right), \quad \mathbf{K}_{2}=\left(\begin{array}{rrr}
0 & 0 & -1 \\
-1 & 0 & 0 \\
0 & 1 & 0
\end{array}\right), \\
\mathbf{K}_{3} & =\left(\begin{array}{rrr}
0 & 1 & 0 \\
-1 & 0 & 0 \\
0 & 0 & 1
\end{array}\right),
\end{aligned}
$$

where the subscript indicates each setup in Fig. 2.

The measurements were made in the room temperature $\left(20^{\circ} \mathrm{C}\right)$. The intensities of the magnetic field applied during the measurements are summarized in Table 2. Figure 3 shows the example of output $\mathbf{M}$ in one of the experiments. We can confirm the linearity between the input magnetic field and output readings. Correlation coefficients for them are larger than 0.999 for all setups of the experiments.

The determined angles are shown in Tables 3 and 4. Obtained angles seem to be determined stably for the range- 1 , 2 and 3 , but some of the angles for the range- 0 are significantly different from the other cases (see $\phi_{Y}$ in Table 3 and $\varphi_{Y}$ in Table 4). This is probably due to worse signal to noise ratios for range-0, i.e., the applied magnetic field for the experiments for range- 0 was not much larger than the noise, which is originated from the external field, up to $2 \mathrm{nT}$. We therefore employ the results for ranges 1,2 and 3 in Tables 3 and 4 to find relative angles between the axes of the sensor (and also the coil). The result is shown in Table 5. All the angles between the axes are in the range of $90 \pm 0.4$ degrees.

Sensitivities are also calculated for each range using the average angles (Tables 3 and 4). Obtained sensitivities 
Table 3. Obtained angles for sensor-mirror alignment (see Fig. 1(a)). Unit is degree.

\begin{tabular}{lllllll}
\hline & $\phi_{X}$ & $\phi_{Y}$ & $\phi_{Z}$ & $\theta_{X}$ & $\theta_{Y}$ & $\theta_{Z}$ \\
\hline range-0 & 0.376 & 0.023 & -0.373 & 0.345 & 0.359 & -0.263 \\
range-1 & 0.339 & -0.032 & -0.364 & 0.496 & 0.333 & -0.031 \\
range-2 & 0.337 & -0.036 & -0.379 & 0.469 & 0.319 & -0.034 \\
range-3 & 0.309 & -0.035 & -0.387 & 0.478 & 0.320 & -0.060 \\
\hline Average of range-1,2,3 & 0.328 & -0.035 & -0.377 & 0.481 & 0.324 & -0.041 \\
\hline
\end{tabular}

Table 4. Obtained angles for coil-mirror alignment (see Fig. 1(b)). Unit is degree.

\begin{tabular}{llrrrrl}
\hline & $\varphi_{X}$ & \multicolumn{1}{l}{$\varphi_{Y}$} & \multicolumn{1}{c}{$\varphi_{Z}$} & \multicolumn{1}{c}{$\lambda_{X}$} & \multicolumn{1}{l}{$\lambda_{Y}$} & \multicolumn{1}{c}{$\lambda_{Z}$} \\
\hline range-0 & 0.000 & 0.060 & 0.065 & -0.099 & 0.338 & -0.133 \\
range-1 & 0.175 & -0.043 & -0.036 & 0.140 & 0.321 & -0.290 \\
range-2 & 0.168 & -0.051 & -0.049 & 0.131 & 0.308 & -0.295 \\
range-3 & 0.169 & -0.046 & -0.050 & 0.129 & 0.324 & -0.306 \\
\hline Average of range-1,2,3 & 0.171 & -0.047 & -0.045 & 0.133 & 0.318 & -0.297 \\
\hline
\end{tabular}

Table 5. Angle between the axes. Unit is degree.

\begin{tabular}{llll}
\hline & $\angle X Y$ & $\angle Y Z$ & $\angle Z X$ \\
\hline MGF-S & 89.61 & 89.73 & 89.89 \\
Coil & 89.84 & 89.98 & 89.97 \\
\hline
\end{tabular}

Table 6. Sensitivity [nT/digit].

\begin{tabular}{llll}
\hline & $A_{X}$ & $A_{Y}$ & $A_{Z}$ \\
\hline range-0 & $0.00229 \pm 0.000002$ & $0.00232 \pm 0.000004$ & $0.00228 \pm 0.000005$ \\
range-1 & $0.00917 \pm 0.000001$ & $0.00932 \pm 0.000003$ & $0.00914 \pm 0.000002$ \\
range-2 & $0.03681 \pm 0.000844$ & $0.03725 \pm 0.000022$ & $0.03656 \pm 0.000013$ \\
range-3 & $2.088 \pm 0.0012$ & $2.122 \pm 0.0012$ & $2.086 \pm 0.0006$ \\
\hline
\end{tabular}

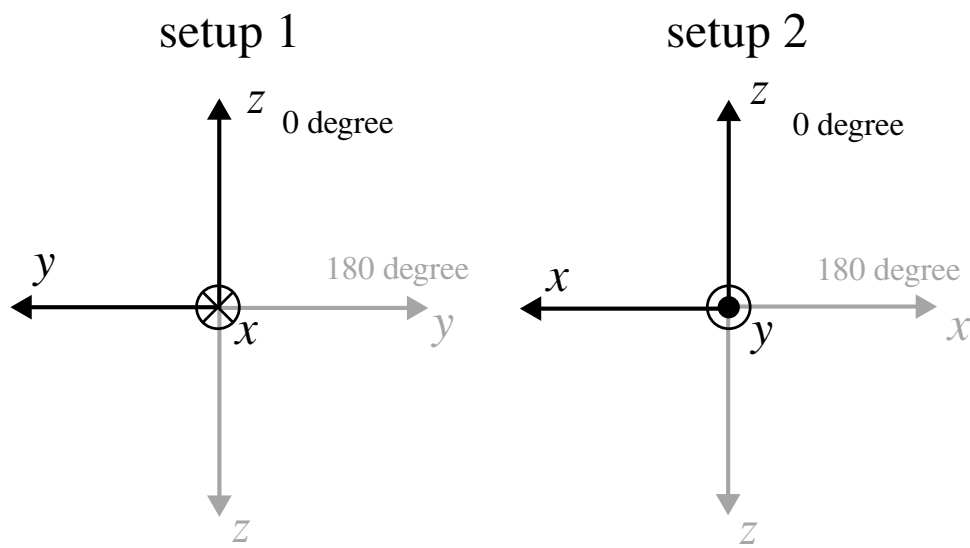

Fig. 4. Sensor coordinates in experiments of zero-offsets.

are summarized in Table 6. The sensitivities were well determined within $0.2 \%$ error.

\subsection{Temperature dependences of offset and relative sensitivity}

Determining zero-offset in Eq. (2) requires a new set of experiments: it is necessary to operate the magnetometer under almost zero magnetic field environment. We design the experiment to determine zero-offset of each sensor axis together with obtaining temperature dependences of zerooffset and relative sensitivity, the ratio of sensitivity to the reference sensitivity, as shown below. Temperature depen- dences should be evaluated because the operating temperature of the MGF-S in the lunar orbit is expected to vary by about 60 degrees $\left(-30 \sim 30^{\circ} \mathrm{C}\right)$.

Zero-offset may be determined by measuring magnetic field under almost zero magnetic field conditions. This may be done using a shield cylinder covering the sensor in the Helmholtz coil system. MGF-S was set as shown in Fig. 4 while determining the offset of the $z$ component; the average of the four readings is the zero-offset value for the $z$ component. The experiments were done for all the ranges by controlling the temperature stepwise from $-35 \sim 33^{\circ} \mathrm{C}$. 


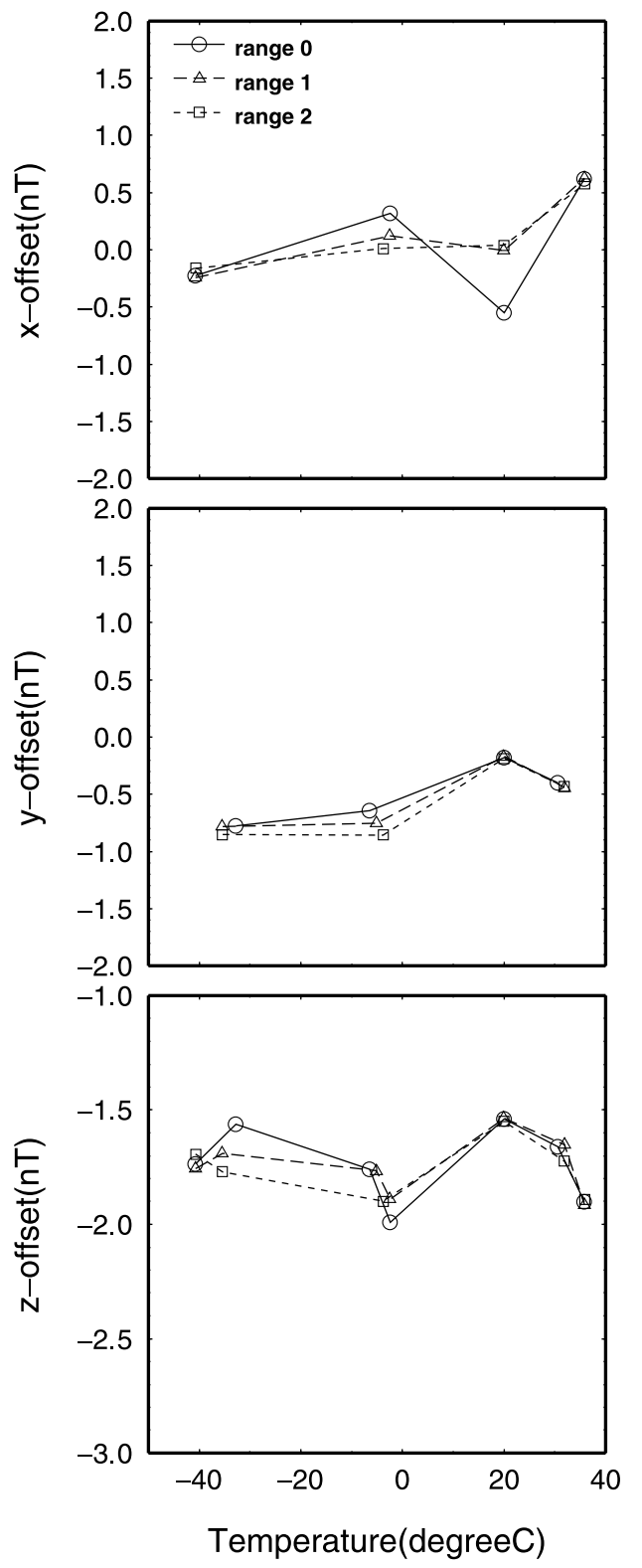

Fig. 5. Temperature dependence of the offset.

An example of the result is shown in Fig. 5. The results for range- 3 are not shown in the figure because the resolution of this mode is low. It is seen that the offset does not depend systematically on temperature. Averages of the offset for ranges 0,1 and 2 are shown in Table 7 .

Variation of sensitivity with respect to temperature is also examined for range-0, which is mainly used for observations while SELENE is orbiting the moon. Because relative change of the reading from the magnetometer in different temperature is essential, we employed only one setup of the relative directions of the magnetometer and the coil system. It is sufficient for the present purpose if the applied field is measured by all the sensors, i.e., the MGF-S was set in the cooled box oblique to the applied field. In the experiment, $-60 \mathrm{nT}, 0 \mathrm{nT}$, and $60 \mathrm{nT}$ of magnetic field is provided in the $x$-direction of the coil system. The temperature range was $-45 \sim 32^{\circ} \mathrm{C}$.

Variation of relative sensitivity against temperature is
Table 7. Averages of the offset in each direction of the MGF-S sensor.

\begin{tabular}{cccc}
\hline & $x(\mathrm{nT})$ & $y(\mathrm{nT})$ & $z(\mathrm{nT})$ \\
\hline range-0 & 0.0 & -0.5 & -1.7 \\
range-1 & 0.1 & -0.5 & -1.8 \\
range-2 & 0.2 & -0.6 & -1.8 \\
\hline
\end{tabular}

shown in Fig. 6. The sensitivity at $14.7^{\circ} \mathrm{C}$ is taken as the reference sensitivity. There are no clear systematic temperature dependences of the sensitivity seen in the results. The maximum difference is about $1.7 \%$, however, the total intensity change is less than $0.34 \%$. This implies that some small mechanical movement of MGF-S might have occurred unexpectedly during the experiment such as, for example, a little change of the MGF-S body position in the cooled box. The uncertainty due to the temperature dependence or range- 0 sensitivity is much less than $0.1 \mathrm{nT}$ for the typical magnetic field of $10 \mathrm{nT}$, and thus the sensitivity change due to temperature do not have to be taken into considerations for analyses of the lunar magnetic field data.

\section{In-flight Calibration Using Sensor Alignment Monitor Coil (SAM-C)}

\subsection{Requirements for in-flight calibrations}

It is a concern that the direction of the magnetometer may change while the satellite is in orbit, especially in case that the satellite experiences large temperature differences due to eclipses. Monitoring of the direction of the sensor is essential to maintain the credibility of the observed magnetic field. We employ the method to monitor the position and alignment of the magnetometer by measuring the known magnetic field, as is similar to the case of calibration of magnetometer in the previous section. In-flight calibrations have been made for the vector magnetometers of satellites by observing the geomagnetic field. For example, the Euler angles describing the rotation of the magnetometer with respect to the satellite were determined with accuracy of better than 4 arcsec for the Oersted magnetometer orbiting the earth (Olsen et al., 2003). Also, observed geomagnetic field during swingby the earth were analyzed to obtain the calibration matrix for the NEAR magnetometer (Anderson et al., 2001) and to renew the alignment angles of the sensors for the Cassini fluxgate magnetometer (Dougherty et al., 2004). As there is no well-known ambient natural magnetic field available in the lunar environment, artificial magnetic fields are generated in the satellite system for in-flight calibration for SELENE. SELENE has the Sensor Alignment Monitor Coil (SAM-C) system to generate known magnetic fields for in-flight calibrations. Note that Cassini has a similar in-flight calibration system called the Science Calibration Subsystem (SCAS, Dougherty et al., 2004).

Because the expected magnetic field in orbit around the moon is small, typically 5-10 nT, the requirement of the accuracy of the direction determination is not as high as that for the satellite magnetometers for geomagnetic field observations. By considering the accuracy of the magnetometer $(0.1 \mathrm{nT})$ and the size of the expected magnetic field, the required accuracy is of order of 0.1 degrees.

SAM-C has two coil systems, SAM-C(A) and SAM- 

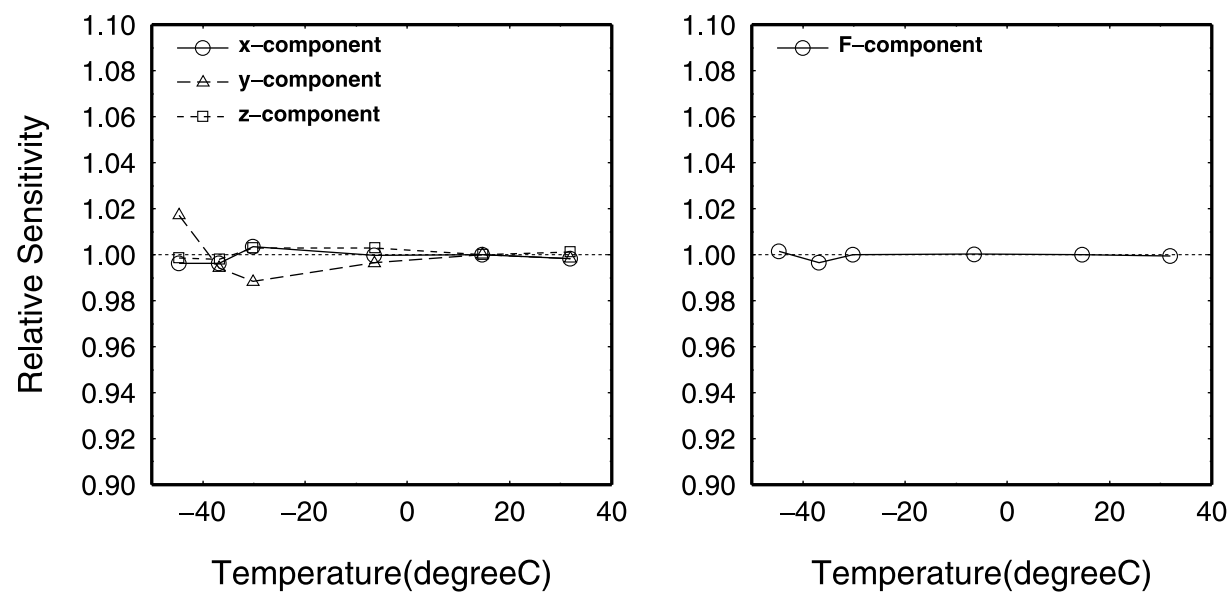

Fig. 6. Temperature dependence of the sensitivity. Ratio to the sensitivity at $14.7^{\circ} \mathrm{C}$ is shown.

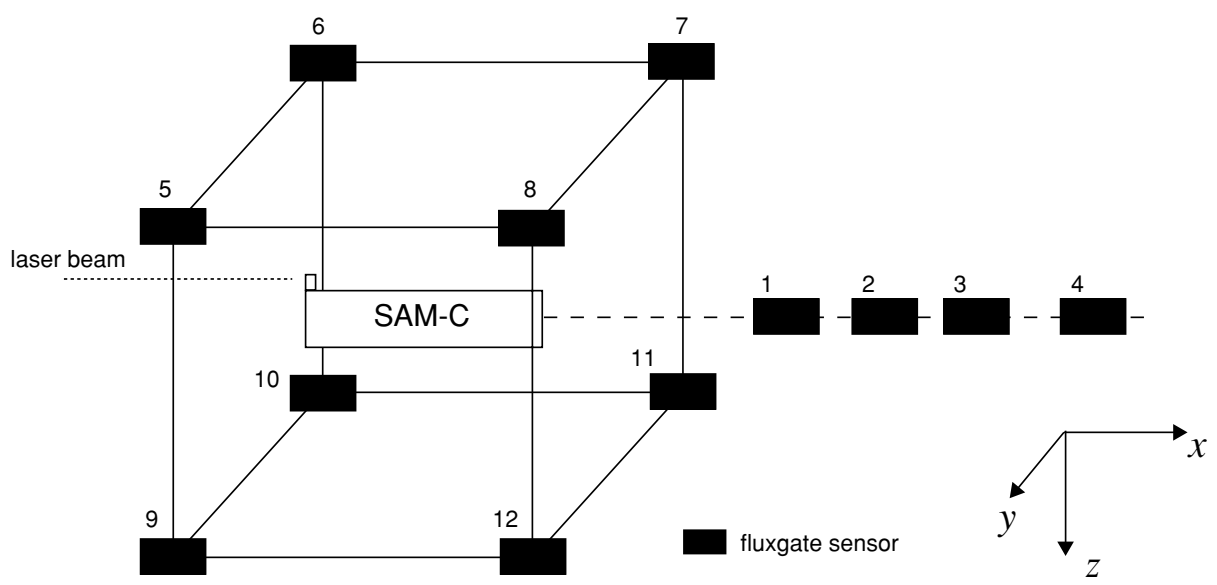

Fig. 7. Setup of the experiment to determine the distributions of the magnetic field generated by SAM-C.

$\mathrm{C}(\mathrm{B})$, in order to generate two linearly independent magnetic fields for in-flight calibrations. The spatial distributions of the magnetic field generated by SAM-C around the position of the magnetometer, about 12 meters away, can be used for accurate calibrations, that is, to determine the position and the rotation angles of the magnetometer with respect to the main body of the satellite. Therefore, 6 unknowns can be determined simultaneously from two independent magnetic field vectors generated by the two coils.

Multiple measurements in a set of calibration can reduce the error of determination of the position and rotation angles. SAM-C is going to generate $1 \mathrm{~Hz}$ triangular wave, which continues with same amplitude for 10 seconds and then decays in 8 seconds. Though the sampling frequency is $32 \mathrm{~Hz}$ and 576 measurements for one set of calibration can be obtained, not all data can be used for the analyses because the magnetic field is not strong enough at the magnetometer position. Nevertheless, a large number of measurements are available for calibrations to reduce the estimation error of the position and alignment. Note that the decaying triangular wave is generated because not only to increase the number of measurements but also to prevent magnetizing the satellite and other instruments by the generated magnetic field.

\subsection{Distribution of magnetic field by SAM-C}

The spatial distribution of the SAM-C generated magnetic field has been determined by an experiment. The experiment was carried out in the magnetic test facility at Tsukuba Space center mentioned in Section 2.1. The set-up of the experiment is as shown in Fig. 7. SAM-C was installed on a horizontal turn table placed in the center of the non-magnetic environment. The magnetic field generated by SAM-C is measured using 12 fluxgate magnetometers installed as shown in the figure. The positions of the magnetometers are listed in Table 8. The direction of SAM-C was changed from 0 to 330 degrees at 30-degree steps in order to increase effective data of different relative positions of the magnetometers to SAM-C. The electric currents given to the coil system were $\pm 2.0 \mathrm{~A}$. Note that the maximum amplitude of the electric currents applied to SAM-C in orbit will be $2.6 \mathrm{~A}$.

The distribution of the magnetic field is expressed using magnetic potential in spherical coordinate,

$V=a \sum_{n=1}^{N} \sum_{m=0}^{n}\left(g_{n}^{m} \cos m \phi+h_{n}^{m} \sin m \phi\right)\left(\frac{a}{r}\right)^{n+1} P_{n}^{m}(\cos \theta)$,

where $(r, \theta, \phi)$ represents standard spherical coordinate 
Table 8. Positions of the magnetometers for SAM-C experiments. The origin of the coordinate is the center of SAM-C. Unit is meter.

\begin{tabular}{cc|cc}
\hline magnetometer & $(x, y, z)$ & magnetometer & $(x, y, z)$ \\
\hline$\# 1$ & $(1.2,0.0,0.0)$ & $\# 7$ & $(1.3225,-1.3375,-1.059)$ \\
$\# 2$ & $(1.6,0.0,0.0)$ & $\# 8$ & $(1.3225,1.3625,-1.060)$ \\
$\# 3$ & $(2.0,0.0,0.0)$ & $\# 9$ & $(-1.3225,1.3375,0.936)$ \\
$\# 4$ & $(3.0,0.0,0.0)$ & $\# 10$ & $(-1.3225,-1.3625,0.937)$ \\
$\# 5$ & $(-1.3225,1.3375,-1.059)$ & $\# 11$ & $(1.3225,-1.3375,0.937)$ \\
$\# 6$ & $(-1.3225,-1.3625,-1.062)$ & $\# 12$ & $(1.3225,1.3625,0.936)$ \\
\hline
\end{tabular}

Table 9. Gauss coefficients determined by the experiments, see Eq. (7). $a=2.1 \mathrm{~m} .+2 \mathrm{~A}$ is given during the experiments.

\begin{tabular}{c|rc|rc}
\hline mode & \multicolumn{2}{|c|}{ SAM-C(A) } & \multicolumn{2}{c}{ SAM-C(B) } \\
\hline$(n, m)$ & \multicolumn{1}{|c|}{$g_{n}^{m}$} & $h_{n}^{m}$ & $g_{n}^{m}$ & $h_{n}^{m}$ \\
\hline$(1,0)$ & 223.0395 & - & 226.5080 & - \\
$(1,1)$ & -155.1038 & -0.3778 & 159.8948 & -5.1260 \\
$(2,0)$ & -4.2798 & - & 5.3552 & - \\
$(2,1)$ & -6.2950 & 1.3071 & 3.9018 & 0.5415 \\
$(2,2)$ & 1.2032 & -0.7165 & -0.5657 & 0.8414 \\
$(3,0)$ & -4.2728 & - & -4.1738 & - \\
$(3,1)$ & -3.9164 & 0.0298 & 4.0347 & -0.1824 \\
$(3,2)$ & 0.9133 & -0.0184 & 0.8367 & -0.0123 \\
$(3,3)$ & -1.1781 & -0.0031 & 1.2309 & -0.0158 \\
$(4,0)$ & 0.3858 & - & 0.3723 & - \\
$(4,1)$ & -0.0314 & -0.0150 & -0.0322 & 0.0785 \\
$(4,2)$ & 0.0592 & -0.0594 & -0.0039 & 0.0860 \\
$(4,3)$ & -0.0191 & -0.0038 & 0.1400 & 0.0220 \\
$(4,4)$ & -0.0429 & 0.0288 & 0.0369 & 0.0069
\end{tabular}
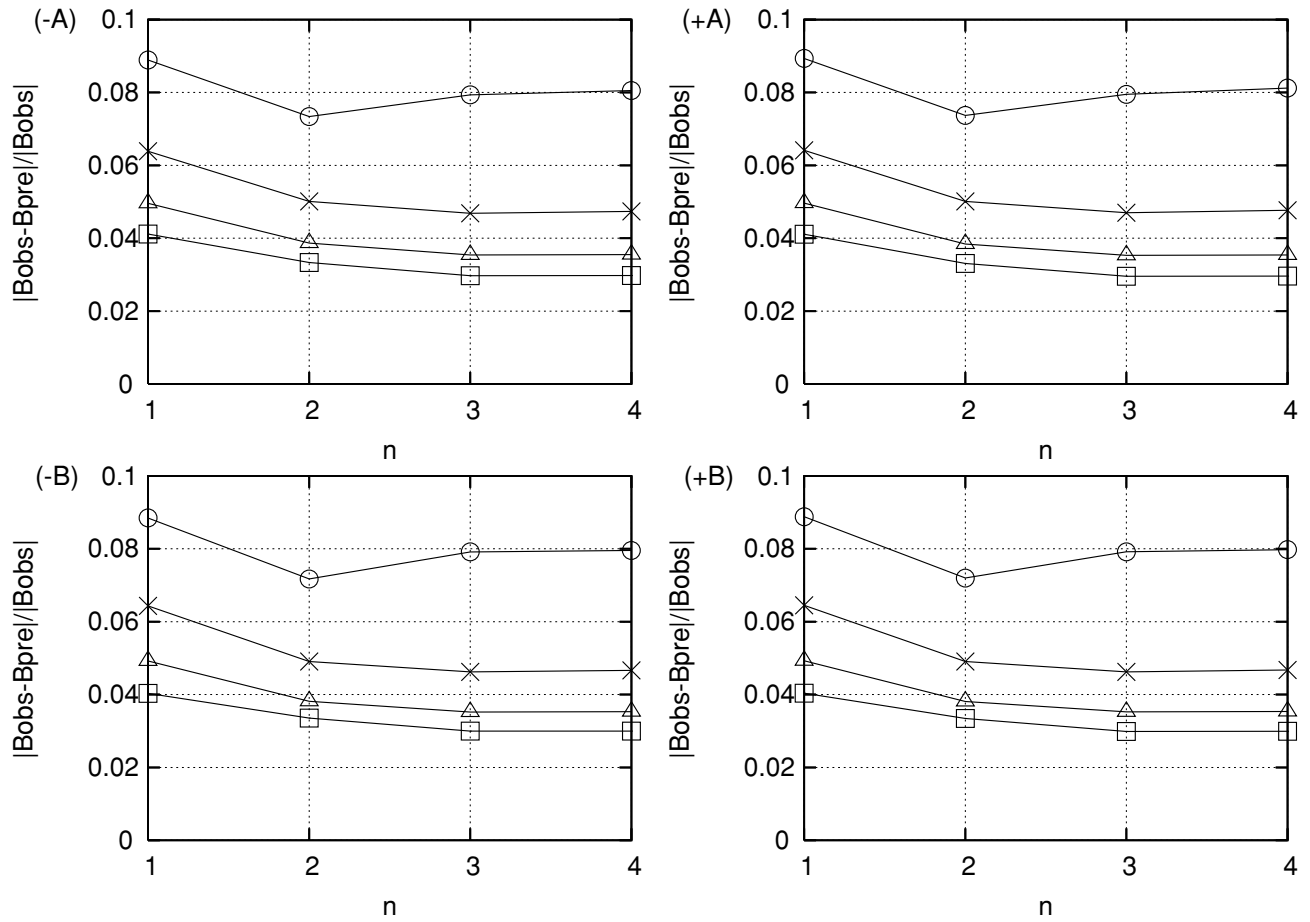

Fig. 8. Misfit of magnetic intensity with respect to truncation of Eq. (7). Open circles, crosses, triangles, and squares represent misfit at magnetometer $1,2,3$ and 4 , respectively.

( $\theta=0$ coincides wish positive $z$ direction), $P_{n}^{m}(\cos \theta)$ is associated Legendre function, $a$ is a certain radius, and $N$ is the truncation degree. The Gauss coefficients $\left(g_{n}^{m}\right.$ and $\left.h_{n}^{m}\right)$ of the magnetic potential with $a=2.1 \mathrm{~m}$ were determined by the experiment; see Table 9 for the case with positive electric current. It is found that the magnetic field at magnetometer position 1, 2, 3, and 4 in Fig. 7 can be expressed with the magnetic potential up to degree and order 4 with accuracy better than 3\% (see Fig. 8). Note that nominal position of the MGF-S in lunar orbit is $(11.724 \mathrm{~m}$, 
$\mathrm{Bx}$
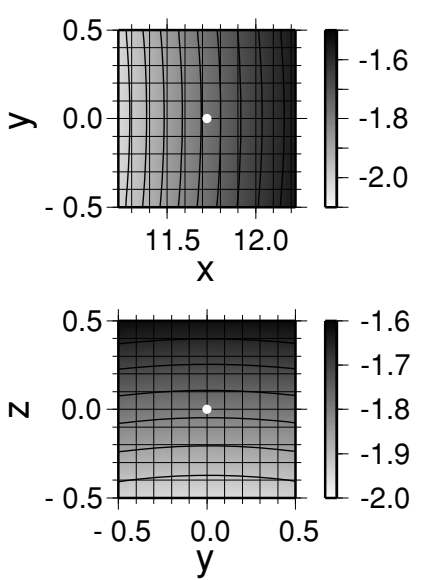

By
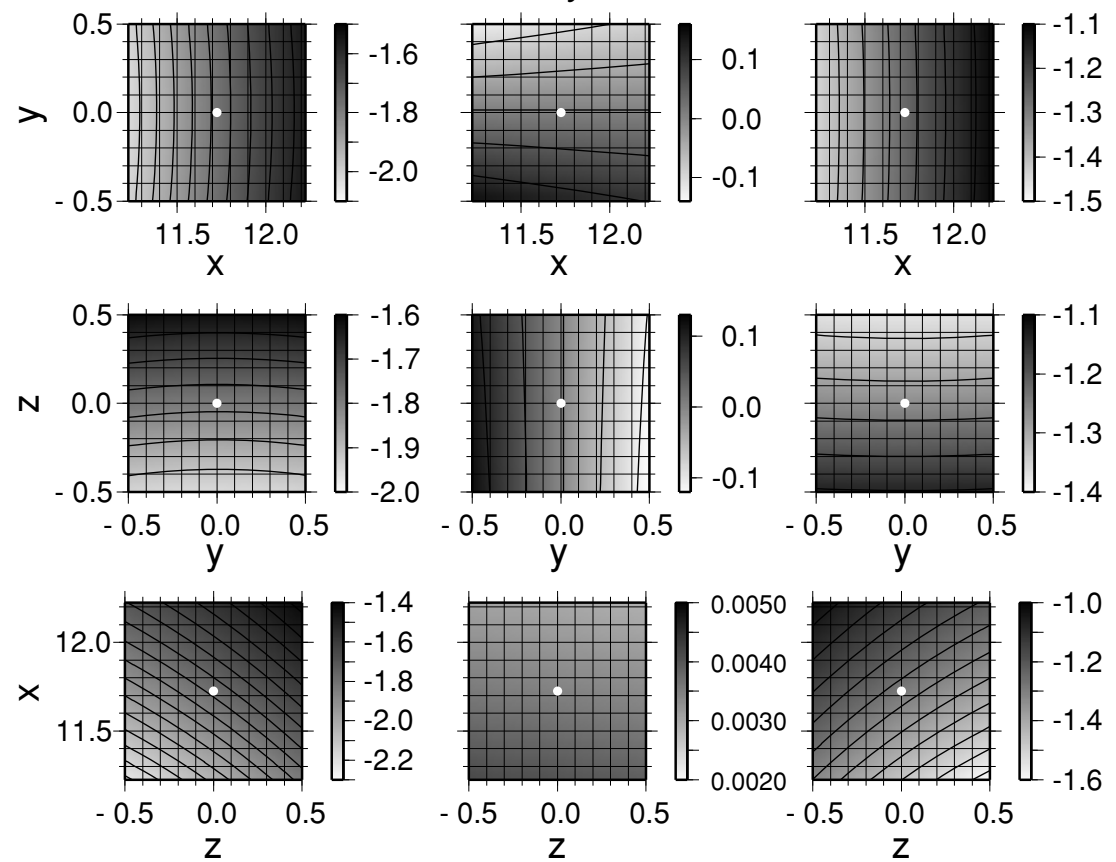

$\mathrm{Bz}$
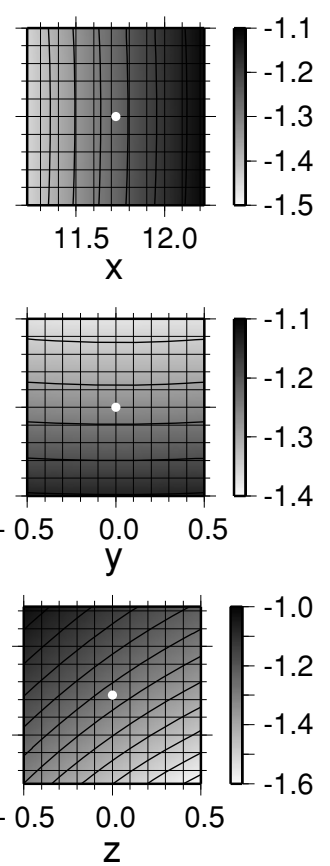

Fig. 9. Magnetic field distribution around the magnetometer position by SAM-C(A). Applied electric current is 2 A.

$0 \mathrm{~m}, 0 \mathrm{~m}$ ) in the supposed coordinate, which is the same as the satellite coordinate, and the contribution of the magnetic potential with $n \geq 5$ at the position is negligibly small (less than $0.01 \mathrm{nT}$ ). The nominal magnetic field at the position of the magnetometer with +2 A electric current by the coil system is, by SAM-C(A)

$$
\begin{aligned}
& B_{x}=-1.79 \mathrm{nT}, B_{y}=0.00 \mathrm{nT}, B_{z}=-1.27 \mathrm{nT}, \\
& F=2.19 \mathrm{nT},
\end{aligned}
$$

and by SAM-C(B),

$$
\begin{aligned}
& B_{x}=1.83 \mathrm{nT}, B_{y}=0.03 \mathrm{nT}, B_{z}=-1.31 \mathrm{nT}, \\
& F=2.33 \mathrm{nT} .
\end{aligned}
$$

The distributions of the magnetic field components around the magnetometer position by SAM-C(A) are shown in Fig. 9.

\subsection{Estimate of the determination error}

We formulate a linear inversion to determine the position and direction of the magnetometer. The matrix for the inverse problem is used to estimate the expected error of determination of the position and alignment (rotation angles).

Magnetic field at position $\mathbf{r}$ in satellite coordinate, $\mathbf{B}_{\mathrm{SAT}}(\mathbf{r})$, may be written as

$$
\mathbf{B}_{\mathrm{SAT}}(\mathbf{r})=\tilde{\mathbf{A}}(\mathbf{r}, N) \mathbf{g}(N),
$$

where $\mathbf{g}(N)$ is column vector composed of Gauss coefficients shown in Table 9, $N$ is the truncation degree of spherical harmonic expansion in Eq. (7), in this case $N=4$, and $\tilde{\mathbf{A}}(\mathbf{r}, N)$ is the matrix having information on the observation point and spherical harmonics.

Suppose the nominal position of the magnetometer is $\mathbf{r}_{0}$ and the direction of the sensors are expressed with rollpitch-yaw type rotation angles $(x-y-z$ type Euler angles)

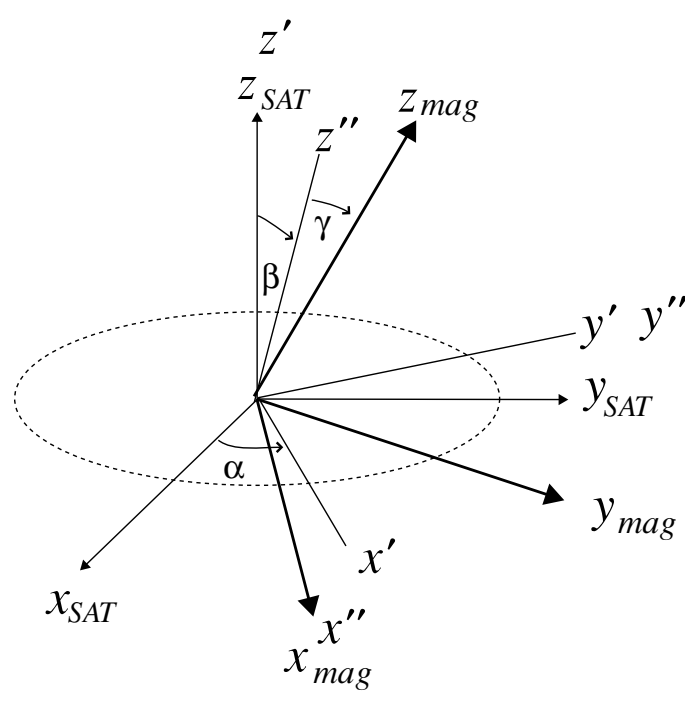

Fig. 10. Definition of the rotation angles.

$(\alpha, \beta, \gamma)$ from the direction of the satellite coordinate (see Fig. 10). At the nominal position, the magnetic field observed by the magnetometer, $\mathbf{B}_{\text {mag }}\left(\mathbf{r}_{0}\right)$, is

$$
\begin{aligned}
\mathbf{B}_{\mathrm{mag}}\left(\mathbf{r}_{0}\right) & =\mathbf{R}(\alpha, \beta, \gamma) \mathbf{B}_{\mathrm{SAT}}\left(\mathbf{r}_{0}\right) \\
& =\mathbf{R}(\alpha, \beta, \gamma) \tilde{\mathbf{A}}\left(\mathbf{r}_{0}, N\right) \mathbf{g}(N),
\end{aligned}
$$

where $\mathbf{R}(\alpha, \beta, \gamma)$ is the rotation matrix

$$
\begin{aligned}
\mathbf{R}(\alpha, \beta, \gamma)= & \left(\begin{array}{ccc}
1 & 0 & 0 \\
0 & \cos \gamma & \sin \gamma \\
0 & -\sin \gamma & \cos \gamma
\end{array}\right)\left(\begin{array}{ccc}
\cos \beta & 0 & -\sin \beta \\
0 & 1 & 0 \\
\sin \beta & 0 & \cos \beta
\end{array}\right) \\
& \cdot\left(\begin{array}{rrr}
\cos \alpha & \sin \alpha & 0 \\
-\sin \alpha & \cos \alpha & 0 \\
0 & 0 & 1
\end{array}\right)
\end{aligned}
$$


The actual position and alignment of magnetometer may be different from the nominal position. Suppose that the actual position of the magnetometer is at $\mathbf{r}_{0}+\Delta \mathbf{r}$ and the Euler angle of the alignment $(\alpha+\Delta \alpha, \beta+\Delta \beta, \gamma+\Delta \gamma)$. Let the deviation of the magnetometer from the nominal position be small and neglect the terms second order in perturbation. Then,

$$
\begin{aligned}
\mathbf{B}_{\mathrm{SAT}}\left(\mathbf{r}_{0}+\Delta \mathbf{r}\right)= & \tilde{\mathbf{A}}\left(\mathbf{r}_{0}+\Delta \mathbf{r}, N\right) \mathbf{g}(N) \\
\approx & \mathbf{B}_{\mathrm{SAT}}\left(\mathbf{r}_{0}\right)+\Delta x \frac{\partial \tilde{\mathbf{A}}\left(\mathbf{r}_{0}\right)}{\partial x} \mathbf{g}(N) \\
& +\Delta y \frac{\partial \tilde{\mathbf{A}}\left(\mathbf{r}_{0}\right)}{\partial y} \mathbf{g}(N)+\Delta z \frac{\partial \tilde{\mathbf{A}}\left(\mathbf{r}_{0}\right)}{\partial z} \mathbf{g}(N) \\
= & \mathbf{B}_{\mathrm{SAT}}\left(\mathbf{r}_{0}\right)+\mathbf{Q}_{x y z} \Delta \mathbf{r},
\end{aligned}
$$

where $\Delta \mathbf{r}=(\Delta x, \Delta y, \Delta z)$ and

$$
\mathbf{Q}_{x y z}=\left(\begin{array}{lll}
\left(\frac{\partial \tilde{\mathbf{A}}\left(\mathbf{r}_{0}\right)}{\partial x} \mathbf{g}\right)_{x} & \left(\frac{\partial \tilde{\mathbf{A}}\left(\mathbf{r}_{0}\right)}{\partial y} \mathbf{g}\right)_{x} & \left(\frac{\partial \tilde{\mathbf{A}}\left(\mathbf{r}_{0}\right)}{\partial z} \mathbf{g}\right)_{x} \\
\left(\frac{\partial \tilde{\mathbf{A}}\left(\mathbf{r}_{0}\right)}{\partial x} \mathbf{g}\right)_{y} & \left(\frac{\partial \tilde{\mathbf{A}}\left(\mathbf{r}_{0}\right)}{\partial y} \mathbf{g}\right)_{y}\left(\frac{\partial \tilde{\mathbf{A}}\left(\mathbf{r}_{0}\right)}{\partial z} \mathbf{g}\right)_{y} \\
\left(\frac{\partial \tilde{\mathbf{A}}\left(\mathbf{r}_{0}\right)}{\partial x} \mathbf{g}\right)_{z} & \left(\frac{\partial \tilde{\mathbf{A}}\left(\mathbf{r}_{0}\right)}{\partial y} \mathbf{g}\right)_{z} & \left(\frac{\partial \tilde{\mathbf{A}}\left(\mathbf{r}_{0}\right)}{\partial z} \mathbf{g}\right)_{z}
\end{array}\right) .
$$

Then, the magnetic field observed by the magnetometer is

$$
\begin{aligned}
\mathbf{B}_{\mathrm{OBS}}= & \mathbf{B}_{\mathrm{mag}}\left(\mathbf{r}_{0}+\Delta \mathbf{r}\right) \\
\approx & \mathbf{R}(\alpha+\Delta \alpha, \beta+\Delta \beta, \gamma+\Delta \gamma) \mathbf{B}_{\mathrm{SAT}}\left(\mathbf{r}_{0}+\Delta \mathbf{r}\right) \\
\approx & \left(\mathbf{R}(\alpha, \beta, \gamma)+\Delta \alpha \frac{\partial \mathbf{R}(\alpha, \beta, \gamma)}{\partial \alpha}\right. \\
& \quad+\Delta \beta \frac{\partial \mathbf{R}(\alpha, \beta, \gamma)}{\partial \beta} \\
& \left.\quad+\Delta \gamma \frac{\partial \mathbf{R}(\alpha, \beta, \gamma)}{\partial \gamma}\right) \mathbf{B}_{\mathrm{SAT}}\left(\mathbf{r}_{0}+\Delta \mathbf{r}\right) \\
& \quad \mathbf{R}(\alpha, \beta, \gamma) \mathbf{B}_{\mathrm{SAT}}\left(\mathbf{r}_{0}\right)+\mathbf{Q}_{\alpha \beta \gamma}\left(\begin{array}{c}
\Delta \alpha \\
\Delta \beta \\
\Delta \gamma
\end{array}\right) \\
& +\mathbf{R}(\alpha, \beta, \gamma) \mathbf{Q}_{x y z}\left(\begin{array}{c}
\Delta x \\
\Delta y \\
\Delta z
\end{array}\right),
\end{aligned}
$$

where

$$
\mathbf{Q}_{\alpha \beta \gamma}=\left(\begin{array}{lll}
\left(\frac{\partial \mathbf{R}}{\partial \alpha} \mathbf{B}_{\mathrm{SAT}}\left(\mathbf{r}_{0}\right)\right)_{x} & \left(\frac{\partial \mathbf{R}}{\partial \beta} \mathbf{B}_{\mathrm{SAT}}\left(\mathbf{r}_{0}\right)\right)_{x} & \left(\frac{\partial \mathbf{R}}{\partial \gamma} \mathbf{B}_{\mathrm{SAT}}\left(\mathbf{r}_{0}\right)\right)_{x} \\
\left(\frac{\partial \mathbf{R}}{\partial \alpha} \mathbf{B}_{\mathrm{SAT}}\left(\mathbf{r}_{0}\right)\right)_{y} & \left(\frac{\partial \mathbf{R}}{\partial \beta} \mathbf{B}_{\mathrm{SAT}}\left(\mathbf{r}_{0}\right)\right)_{y} & \left(\frac{\partial \mathbf{R}}{\partial \gamma} \mathbf{B}_{\mathrm{SAT}}\left(\mathbf{r}_{0}\right)\right)_{y} \\
\left(\frac{\partial \mathbf{R}}{\partial \alpha} \mathbf{B}_{\mathrm{SAT}}\left(\mathbf{r}_{0}\right)\right)_{z} & \left(\frac{\partial \mathbf{R}}{\partial \beta} \mathbf{B}_{\mathrm{SAT}}\left(\mathbf{r}_{0}\right)\right)_{z} & \left(\frac{\partial \mathbf{R}}{\partial \gamma} \mathbf{B}_{\mathrm{SAT}}\left(\mathbf{r}_{0}\right)\right)_{z}
\end{array}\right) .
$$

We may solve for the perturbations if at least two linearly independent magnetic field distributions are available for the analysis. The two independent magnetic fields generated by SAM-C fulfill the condition.

Let the magnetic field by SAM-C(A) be $\mathbf{B}_{\mathrm{SAT}}(\mathrm{A})$ and observed field be $\mathbf{B}_{\mathrm{OBS}}(\mathrm{A})$. Same notations are used for the field by SAM-C(B). Then, the equation may be written as

$$
\Delta \tilde{\mathbf{b}} \equiv\left(\begin{array}{c}
\mathbf{B}_{\mathrm{OBS}}(\mathrm{A}) \\
\ldots \\
\mathbf{B}_{\mathrm{OBS}}(\mathrm{B})
\end{array}\right)-\left(\begin{array}{c}
\mathbf{R B}_{\mathrm{SAT}}(\mathrm{A}) \\
\ldots \\
\mathbf{R B}_{\mathrm{SAT}}(\mathrm{B})
\end{array}\right)=\mathbf{V}\left(\begin{array}{c}
\Delta \alpha \\
\Delta \beta \\
\Delta \gamma \\
\Delta x \\
\Delta y \\
\Delta z
\end{array}\right)
$$$$
\equiv \mathbf{V} \Delta \tilde{\mathbf{q}}
$$

where the matrix $\mathbf{V}$ is written using $\mathbf{Q}_{x y z}$ and $\mathbf{Q}_{\alpha \beta \gamma}$. The matrix may be inverted to obtain $\Delta \tilde{\mathbf{q}}$;

$$
\Delta \tilde{\mathbf{q}}=\mathbf{V}^{-1} \Delta \tilde{\mathbf{b}}
$$

The accuracy of determination of $\Delta \tilde{\mathbf{q}}$ can be estimated using the singular values (eigenvalues) and condition number of the matrix. The condition number of the matrix $\mathbf{V}$ is very large, about 2400 , either with $(\alpha, \beta, \gamma)=(0,0,0)$ or $(0,0,45)$ (unit in degree) if the Gauss coefficients in Table 9 are used (i.e. the electric current for SAM-C is $2.0 \mathrm{~A}$ ). As the $y$-derivatives of $B_{x}$ and $B_{z}$ relative to the others both for SAM-C(A) and SAM-C(B) magnetic field are small, the expected error is very large in this case, see Fig. 9: the components of $\mathbf{Q}_{x y z}$ matrix for SAM-C(A) at the nominal position of the magnetometer are

$\mathbf{Q}_{x y z}=\left(\begin{array}{rr}4.569 \times 10^{-1}-9.909 \times 10^{-4} & 3.248 \times 10^{-1} \\ -9.909 \times 10^{-4}-2.280 \times 10^{-1} & -1.977 \times 10^{-4} \\ 3.248 \times 10^{-1}-1.977 \times 10^{-4} & -2.288 \times 10^{-1}\end{array}\right)$,

where the unit is $n T / m$. The tendency is the same for the matrix for SAM-C(B).

It is essential to determine the direction of the magnetometer at the end of the mast but the position is not essential for scientific purposes. The small $y$-derivatives of the magnetic field components implies that precise determination of the $y$ position of the magnetometer is not necessary for the determination of the alignment (and also for scientific purposes).

Now, let us consider a reduced problem having 5 unknowns,

$$
\begin{aligned}
\mathbf{B}_{\mathrm{OBS}}= & \mathbf{R}(\alpha, \beta, \gamma) \mathbf{B}_{\mathrm{SAT}}\left(\mathbf{r}_{0}\right)+\mathbf{Q}_{\alpha \beta \gamma}\left(\begin{array}{c}
\Delta \alpha \\
\Delta \beta \\
\Delta \gamma
\end{array}\right) \\
& +\mathbf{R}(\alpha, \beta, \gamma) \mathbf{Q}_{x z}\left(\begin{array}{c}
\Delta x \\
\Delta z
\end{array}\right),
\end{aligned}
$$

where

$$
\mathbf{Q}_{x z}=\left(\begin{array}{ll}
\left(\frac{\partial \mathbf{A}(\mathbf{r})}{\partial x} \mathbf{g}\right)_{x} & \left(\frac{\partial \mathbf{A}(\mathbf{r})}{\partial z} \mathbf{g}\right)_{x} \\
\left(\frac{\partial \mathbf{A}(\mathbf{r})}{\partial x} \mathbf{g}\right)_{y} & \left(\frac{\partial \mathbf{A}(\mathbf{r})}{\partial z} \mathbf{g}\right)_{y} \\
\left(\frac{\partial \mathbf{A}(\mathbf{r})}{\partial x} \mathbf{g}\right)_{z} & \left(\frac{\partial \mathbf{A}(\mathbf{r})}{\partial z} \mathbf{g}\right)_{z}
\end{array}\right)
$$

and

$$
\Delta \tilde{\mathbf{b}}=\mathbf{V}_{r}\left(\begin{array}{c}
\Delta \alpha \\
\Delta \beta \\
\Delta \gamma \\
\Delta x \\
\Delta z
\end{array}\right) \equiv \mathbf{V}_{r} \Delta \tilde{\mathbf{q}}_{r}
$$

This may be solved in a least-square sense by

$$
\Delta \tilde{\mathbf{q}}_{r}=\left(\mathbf{V}_{r}^{T} \mathbf{V}_{r}\right)^{-1} \mathbf{V}_{r}^{T} \Delta \tilde{\mathbf{b}} .
$$

The condition numbers for the case with $\alpha=\beta=0$ degree and $\gamma=0$ or 45 degrees are about 17 , and the determination error for each component is estimated as

$$
\Delta x=8.8 \times 10^{-2} \mathrm{~m}, \Delta z=3.7 \times 10^{-1} \mathrm{~m},
$$


and

$$
\begin{aligned}
& \Delta \alpha=1.59 \text { degree, } \Delta \beta=3.86 \text { degree, } \\
& \Delta \gamma=2.22 \text { degree, }
\end{aligned}
$$

when the electric current for SAM-C is $2.0 \mathrm{~A}$.

The estimated error depends linearly on the magnetic field gradient of the SAM-C generated magnetic field. Because the maximum amplitude of the planned SAM-C electric current in the lunar orbit is $2.6 \mathrm{~A}$, the estimated accuracy in the above is reduced by $1 / 1.3$. The estimated error is not small enough if only one measurement is used to determine the quantities. However, as was mentioned previously, $1 \mathrm{~Hz}$ triangular waves are applied and measurements are made with $32 \mathrm{~Hz}$. Because triangular waves are given, not all the data may be used for the in-flight calibration. Probably, taking half of the total 576 measurements, i.e. 288, in one set of calibration is a fair estimate of the effective number of measurements. The estimated error is reduced by $1 / \sqrt{288}$ and the estimate of determination error for one set of calibration is

$$
\Delta x=4.0 \times 10^{-3} \mathrm{~m}, \Delta z=1.6 \times 10^{-2} \mathrm{~m},
$$

and

$$
\begin{aligned}
& \Delta \alpha=6.9 \times 10^{-2} \text { degree, } \Delta \beta=1.8 \times 10^{-1} \text { degree, } \\
& \Delta \gamma=1.0 \times 10^{-1} \text { degree, }
\end{aligned}
$$

in the orbit condition. The direction of the magnetometer can be determined with accuracy of order 0.1 degree, which is sufficient for the magnetic field measurements around the moon.

\section{Summary}

The ground calibration of SELENE magnetometer has been made by a series of experiments. The linearities of the sensors are confirmed and sensitivity for all ranges are determined. The sensors are orthogonal to each other within 0.4 degree, and further correction can be made using the relative angles of the sensors. The offsets and sensitivities do not show any systematic dependencies on temperature. Thus we conclude that the accuracy of MGF-S is about $0.1 \mathrm{nT}$.

The magnetic field distributions generated by SAM-C, which is used for in-flight calibrations, are determined by the experiments. An electric current of 2 A produces a magnetic field of about $2 \mathrm{nT}$ at the position of the magnetometer, which is $12 \mathrm{~m}$ away from SAM-C. A formulation to determine the relative position and angles of the magnetometer with respect to the main body of the satellite is given and expected error to determine the quantities are estimated. The estimated error of the direction determination is of order 0.1 degree. Therefore, it is expected that the LMAG data after appropriate corrections using ground and in-flight calibration information have sufficient accuracy to discuss the lunar magnetic anomalies, the interior of the moon and so on.

Acknowledgments. We would like to thank Hirokuni Oda at Advanced Industrial Science and Technology and Yuhji Yamamoto at University of Liverpool for their assistance for the experiments. We are grateful to T. Omoto at Meisei Electric Co., Ltd. and Y. Takeuchi at Nippi Corporation for various help.

\section{References}

Anderson, B. J., L. J. Zanetti, D. H. Lohr, J. R. Hayes, M. H. Acuña, C. T. Russell, and T. Mulligan, In-flight calibration of the NEAR magnetometer, IEEE Trans. on Geoscience and Remote Sensing, 39, 907-917, 2001.

Dougherty, M. K., S. Kellock, D. J. Southwood, A. Balogh, E. J. Smith, B. T. Tsurutani, B. Gerlach, K.-H. Glassmeier, F. Gleim, C. T. Russell, G. Erdos, F. M. Neubauer, and S. W. H. Cowley, The Cassini magnetic field investigation, Space Science Rev., 114, 331-383, 2004.

Dyal, P., C. W. Parkin, and W. D. Daily, Lunar electrical conductivity and magnetic permeability, Proc. Lunar Sci. Conf. 6th, 2909-2926, 1975.

Dyal, P., C. W. Parkin, and W. D. Daily, Structure of the lunar interior from magnetic field measurements, Proc. Lunar Sci. Conf. 7th, 3077-3095, 1976.

Halekas, J. S., D. L. Mitchell, R. P. Lin, S. Frey, L. L. Hood, M. H. Acuña, and A. B. Binder, Mapping of crustal magnetic anomalies on the lunar near side by the Lunar Prospector electron reflectometer, J. Geophys. Res., 106, 27841-27852, 2001.

Hobbs, B. A., The inverse problem of the moon's electrical conductivity, Earth Planet. Sci. Lett., 17, 380-384, 1973.

Hood, L. L., Magnetic field and remanent magnetization effects of basinforming impacts on the moon, Geophys. Res. Lett., 14, 844-847, 1987.

Hood, L. L. and G. Schubert, Lunar magnetic anomalies and surface optical properties, Science, 208, 49-51, 1980.

Hood, L. L., P. J. Coleman, Jr., and D. E. Wilhelms, The moon: sources of the crustal magnetic anomalies, Science, 204, 53-57, 1979.

Hood, L. L., F. Herbert, and C. P. Sonett, The deep lunar electrical conductivity profile: Structural and thermal inferences, J. Geophys. Res., 87, 5311-5326, 1982.

Hood, L. L., D. L. Mitchell, R. P. Lin, M. H. Acuña, and A. B. Binder, Initial measurements of lunar induced magnetic dipole moment using Lunar Prospector magnetomter, Geophys. Res. Lett., 26, 2327-2330, 1999.

Hood, L. L., A. Zakharian, J. Halekas, D. L. Mitchell, R. P. Lin, M. H. Acuña, and A. B. Binder, Initial mapping and interpretation of lunar crustal magnetic anomalies using Lunar Prospector magnetometer data, J. Geophys. Res., 106, 27825-27839, 2001.

Kurata, M., H. Tsunakawa, Y. Saito, H. Shibuya, M. Matsushima, and H. Shimizu, Mini-magnetosphere over the Reiner Gamma magnetic anomaly region on the moon, Geophys. Res. Lett., 32, L24205, doi: 10.1029/2005GL024097, 2005.

Lee, D. C., A. N. Halliday, G. A. Snyder, and L. A. Taylor, Age and origin of the moon, Science, 278, 1098-1103, 1997.

Lohr, D. A., L. J. Zanetti, B. J. Anderson, T. A. Potemra, J. R. Hayes, R. E. Gold, R. M. Henshaw, F. F. Mobrey, and D. B. Holland, NEAR magnetic field investigation, instrumentation, spacecraft magnetic and data access, Space Science Rev., 82, 225-281, 1997.

Olsen, N., L. Toeffiner-Clausen, T. J. Sabaka, P. Brauer, J. M. G. Merayo, J. L. Joergensen, J.-M. Léger, O. V. Nielsen, F. Primdahl, and T. Risbo, Calibration of the Oersted vector magnetometer, Earth Planets Space, 55, 11-18, 2003.

Righter, K., Does the moon have a metallic core? Constraints from giant impact modeling and siderophile elements, Icarus, 158, 1-13, 2002.

Risbo, T., P. Brauer, J. M. G. Merayo, O. V. Nielsen, J. R. Petersen, F. Primdahl, and I. Richter, Oersted pre-flight magnetometer calibration mission, Meas. Sci. Technol., 14, 674-688, 2003.

Runcorn, S. K., The formation of the lunar core, Geochim. Cosmochim. Acta, 60, 1205-1208, 1996.

Russell, C. T., P. J. Coleman, Jr., and B. E. Goldstein, Measurements of the lunar induced magnetic moment in the geomagnetic tail: Evidence for a lunar core?, Proc. Lunar Planet. Sci. Conf., 12, 831-836, 1981.

Toyoshima, M., H. Shibuya, M. Matsushima, H. Shimizu, and H. Tsunakawa, Equivalent source mapping of the lunar crustal magnetic field using ABIC, Earth Planets Space, 60, this issue, 365-373, 2008.

Yamamoto, T., S. Kokubun, and GEOTAIL/PLANET-B MGF Team, Ground calibration of high-sensitivity magnetometers for spacecraft use, Uchu Kagaku Kenkyuusho Houkoku, 88, 1-24, 1996.

H. Shimizu (e-mail: shimizu@eri.u-tokyo.ac.jp), F. Takahashi, N. Horii, A. Matsuoka, M. Matsushima, H. Shibuya, and H. Tsunakawa 